Research Article

\title{
Echocardiography in Confirmed and Highly Suspected Symptomatic COVID-19 Patients and Its Impact on Treatment Change
}

\author{
Nadia Benyounes $\mathbb{D}^{1},{ }^{1}$ Clélie Van Der Vynckt, ${ }^{1}$ Séverine Tibi, ${ }^{2}$ Alexandra Iglesias, ${ }^{3}$ \\ Laurence Salomon, ${ }^{4}$ Olivier Gout ${ }^{5}$ and Thierry Tibi ${ }^{1}$ \\ ${ }^{1}$ Cardiology Unit, Rothschild Foundation Hospital, Paris, France \\ ${ }^{2}$ British Library, London, UK \\ ${ }^{3}$ Alsacienne School, Paris, France \\ ${ }^{4}$ Clinical Research Department, Rothschild Foundation Hospital, Paris, France \\ ${ }^{5}$ Neurology Department, Rothschild Foundation Hospital, Paris, France \\ Correspondence should be addressed to Nadia Benyounes; nbenyounes@for.paris
}

Received 28 May 2020; Revised 22 August 2020; Accepted 5 September 2020; Published 16 September 2020

Academic Editor: Paolo Severino

Copyright (c) 2020 Nadia Benyounes et al. This is an open access article distributed under the Creative Commons Attribution License, which permits unrestricted use, distribution, and reproduction in any medium, provided the original work is properly cited.

\begin{abstract}
Background. COVID-19 interacts at multiple levels with the cardiovascular system. The prognosis of COVID-19 infection is known to be worse for patients with underlying cardiovascular diseases. Furthermore, the virus is responsible for many cardiovascular complications. Myocardial injury may affect up to $20 \%$ of the critically ill patients. However, echocardiography's impact on the management of patients affected by COVID-19 remains unknown. Objectives. To explore echocardiography's impact on the management of COVID-19 patients. Methods. This study was conducted from March $24^{\text {th }}$ to April 14 ${ }^{\text {th }}, 2020$, in a single center at Adolphe de Rothschild Foundation Hospital, Paris, France. All consecutive inpatients with laboratory and/or CT COVID-19 diagnosis were included in this study. Patients' characteristics (clinical, biological, and imaging) and treatment change induced by echocardiography were collected and analyzed. Patients with and without treatment change induced by echocardiography were compared. Results. A total of 56 echocardiographies in 42 patients with highly suspected or confirmed COVID-19 were included in the final analyses. The median age was 66 (IQR 60.5-74). Echocardiography induced a treatment change in 9 cases $(16 \%)$. The analyzed clinical data were not associated with any treatment change induced by echocardiography. D-dimer and Troponin levels were the only biological predictors of the induced treatment change. On echocardiography, higher systolic pulmonary arterial pressure and documented cardiac thrombi were associated with treatment changes in these patients. Conclusions. Echocardiography may be useful for the management of selected COVID-19 patients, especially those with elevated D-Dimer and Troponin levels, in up to $16 \%$ of patients.
\end{abstract}

\section{Introduction}

First seen in Wuhan, China, at the end of 2019, a novel coronavirus, named severe acute respiratory syndrome coronavirus 2 (SARS-CoV-2), became responsible for a pandemic acute respiratory disease around the world, a term coined by the World Health Organization (WHO) COVID19, "Coronavirus Disease 2019."
At the time of writing, COVID-19 has affected a total of 2,374,141 individuals in 185 countries and resulted in 164,716 deaths worldwide. Eighty days later, 11,850,886 patients are affected in 188 countries and 544,726 patients died [1].

It has been documented that preexisting cardiovascular disease (CVD) predisposes to COVID-19 infection and worsens COVID-19 patients' outcome [2-4]. 
Furthermore, at least $20 \%$ of severely ill COVID-19 patients experience cardiac injury [5]. In Chinese experience, patients with no comorbidities have a case fatality rate (CFR) of $0.9 \%$ while those with CVD, hypertension (HTN), and diabetes mellitus have CFR of, respectively, 10.5\%, 6\%, and $7.3 \%[6]$.

As it is sometimes difficult to differentiate cardiac from respiratory acute illness, transthoracic echocardiography (TTE) is a very useful tool in routine clinical practice. However, in the setting of COVID-19, clinicians delivering cardiovascular care are at risk of developing the illness or becoming vectors for the infection. This is especially the case for the sonographers and cardiologists who acquire TTE and mostly transesophageal (TEE) images because of a close contact, exposure, and the duration of the echocardiographic examinations. This is why, systematic TTE and TEE are not recommended in the setting of COVID-19, unless urgently or emergently necessary [7]. An initial step in reducing the number of echocardiographies in these patients would be to assess the indications rigorously. Cardiovascular considerations for patients, health care workers, and health systems during the COVID-19 pandemic have been published very recently [7].

However, in the real-life practice, we are very committed to this part of the medical practice [8-10] and we often have to adapt the recommendations and guidelines to the circumstances. This is why we had to perform TTEs/TEEs on COVID-19 patients admitted to our institution.

The objective of this study was to collect clinical, biological, and echocardiographic data of COVID-19 patients who were admitted to various departments of our institution and who underwent echocardiography, to find out the reasons of TTE/TEE examinations in these patients and to assess the impact of TTE/TEE on their management.

\section{Materials and Methods}

2.1. Patients and Echocardiographic Examinations. By the end of March 2020, our hospital opened dedicated departments to COVID-19 patients' care. During their hospitalization in these departments, the patients who underwent echocardiography at the discretion of their physicians were prospectively included in the study.

TTE and TEE were performed on confirmed or highly suspected COVID-19 patients admitted to our institution, by dedicated teams of one cardiologist and one nurse every time. Cardiologists and nurses were equipped with the locally advised personal protective equipment (PPE). TTEs were fast echos, all the measurements being made offline, unless emergently necessary for patients' management. Electrocardiographic monitoring was not obtained during the examination. Two-to-three-second clips were acquired of each of these views: parasternal long axis, parasternal short axis (at least at midpapillary axis), apical 4-chamber, apical 2-chamber views, and subxiphoid view (including inferior vena cava analysis throughout the respiratory cycle on M-mode imaging or clip or both).
Pulsed-wave Doppler of the mitral inflow at the level of valve leaflet tips was used to measure the peak early (E-wave) and late (A-wave) diastolic flow velocities and calculate the E/A ratio. Pulsed-wave Doppler tissue imaging was performed at the lateral and septal mitral annulus to obtain average peak longitudinal early diastolic annular $\left(e^{\prime}\right)$ velocity which was used to calculate the $E / e^{\prime}$ ratio and at the tricuspid annulus. Peak velocity of the tricuspid regurgitation was determined using continuous-wave Doppler.

These clips and images allowed an analysis of the cardiac anatomy and the offline calculations (left ventricular ejection fraction, E/e' ratio, and pulmonary pressures).

A dedicated EPIQ7 ultrasound system was used, brought each time at patients' bedsides, and fully cleaned and decontaminated afterwards.

As the aim of this study was to address the impact of the echocardiographic examinations on patients' treatments, all the performed echocardiographies were included in the study.

Of note, in our hospital as in most French hospitals, echocardiographies are performed by cardiologists and not sonographers.

The very first echocardiographies prospectively performed in our hospital on confirmed or highly suspected COVID-19 patients are reported in this article.

This study was submitted to our institution's ethical comity.

2.2. Treatment Change. Cardiologists' recommendations and treatment modifications induced by echocardiography were prospectively collected. These interventions due to echocardiography could be the introduction of diuretics, of anticoagulation, the thrombolysis of pulmonary embolism after TEE visualization of thrombus in the pulmonary artery in an intubated instable patient, for example.

These interventions were part of the routine clinical practice.

2.3. Data Collection. Epidemiological, demographic, clinical, laboratory, and outcome data were prospectively extracted from electronic medical records. Treatment modifications induced by echocardiography were prospectively collected by the cardiologist himself.

2.4. Statistics. Continuous data were expressed as median (interquartile range [IQR]) values and were compared using the $t$-test, the Mann-Whitney $U$ test, or Kruskal-Wallis $\mathrm{H}$ test, as appropriate. Categorical data were expressed as proportions. Pearson's chi-squared test and Fisher's exact test were used for the comparison of categorical variables, as appropriate. For all the statistical analyses, $P<0.05$ was considered to be statistically significant. Statistical analyses were performed using STATA ${ }^{\circledR}$ software, version 13 (StataCorp LP, College Station, TX, USA). 


\section{Results}

From March $24^{\text {th }}$ to April $14^{\text {th }}, 2020,50$ TTEs, 5 TTE + TEE, and 1 TEE were performed on 42 confirmed or highly suspected COVID-19 patients admitted to our institution. In 4 cases $(7.1 \%)$, nasopharyngeal swab was positive for SARSCoV-2. In 25 cases, nasopharyngeal swab was positive for SARS-CoV-2 and chest CT scan was typical of COVID-19 (44.6\%). In 22 cases (39.3\%), COVID-19 was highly suspected on chest CT, which showed typical abnormalities of the disease. Finally, in 5 cases, CT showed suspect but not typical images of COVID-19 (8.9\%).

Two of the included patients had three TTEs and ten patients had 2 TTEs during the study period, as indicated by the practitioner in charge. The indications for TTE/TEE are summarized in Table 1.

Table 1 describes the clinical characteristics of the included patients. The median age of the patients was 66 years and $32.1 \%$ were female. Inaugural symptoms were respiratory in $62.5 \%$ of cases and neurological in $28.6 \%$. A total of $25 \%$ of the patients were receiving supplemental oxygen at baseline, $58.9 \%$ were intubated, and $16.1 \%$ did not need oxygen therapy at baseline. The median body mass index was $28.7 \mathrm{Kg} / \mathrm{m}^{2}$.

Pulmonary embolism was documented in $17.9 \%$ of the whole series and cardiac thrombus in 3 patients (5.4\%). Figures 1(a) and 1(b) show a right atrial thrombus seen on TTE and Figures 2(a) and 2(b) show a right atrial thrombus on TTE and TEE, respectively.

A treatment change was induced by the performed echocardiography in 9 cases (16\%). These patients' characteristics and the nature of treatment change are summarized in Table 2.

Table 3 describes the biological characteristics of our patients. Among the analyzed biological parameters, D-dimer levels were significant predictors of treatment change induced by echocardiography, and so were the Troponin levels (see Table 3).

Creatinine, NT-pro BNP, fibrinogen, and blood type were not associated with echocardiography induced treatment change.

Apart from these 9 patients, there were other substantial abnormalities documented by echocardiography. In one male patient with stroke and bilateral renal infarctions, who was in atrial fibrillation, sludge was documented in the left atrial appendage by TEE. In a few other cases, TTE helped the physicians in the decision to not proceed to a vascular filling in the setting of hypotension, for example, because of high left ventricular filling pressures. However, these abnormalities did not directly change the treatment.

\section{Discussion}

In this report, the indications for TTEs were in accordance with the known cardiovascular complications of COVID-19. Hence, cardiovascular complications are becoming a major threat to this disease. After the initiation of our study, ASE Statement on Protection of Patients and Echocardiography Service Providers has been published [11].
We wish to discuss our results according to the available data in this field.

4.1. Prothrombotic State and Pulmonary Embolism. In 9 cases (16.1\%), the indication for TTE was the suspicion or the documentation of pulmonary embolism. Indeed, pulmonary embolism has been reported in COVID-19 patients [12], and $\mathrm{D}$-dimer levels have been identified as prognostic markers in infected patients in China $[4,13]$. Furthermore, our results identify D-dimer levels as a marker of treatment change induced by echocardiography. Hence, the median D-dimer level was $20000 \mathrm{ng} / \mathrm{ml}$ (IQR 2690-20000) in the group with treatment change versus 2770 (IQR 1350-9330) in the group without echocardiography induced treatment change $(P=0.0142)$. Of note, in 10 cases $(17.9 \%)$, CT scan has documented pulmonary embolism in this study. The association between COVID-19 induced pneumonia and pulmonary embolism has been questioned [12]. Although our study did not aim to answer this question, the results are in line with a possible association between these two pulmonary pathologies.

In 2 cases, 1 confirmed and 1 suspected COVID-19 patients, a right atrial thrombus was documented by echocardiography (see Figures 1 and 2). Patient 2 had thrombolysis for threatening pulmonary embolism.

Median estimated PAPs in our patients was $38 \mathrm{mmHg}$ (IQR 31-43), with a trend to higher PAPs in the patients with echocardiographic induced treatment change $(P=0.0549)$.

4.2. Ischemic Stroke. An abnormal coagulation state has been documented in Chinese patients, 3 of whom have been described with multiple cerebral infarctions and antiphospholipid antibodies [14].

In 10 cases, the initial manifestation of COVID-19 infection in our study was ischemic stroke. In 1 case, bilateral kidney infarctions were also documented, the patient being in atrial fibrillation, under anticoagulant treatment, and having left atrial appendage sludge on TEE.

4.3. Left Ventricular Systolic Function and Filling Pressures. In the general population, echocardiography is a routine test that influences therapeutic decision-making [15]. Left ventricular systolic function is of major importance in COVID-19 patients' care.

The American College of Cardiology advises restricting echocardiography in COVID-19 patients with myocardial injury or elevated natriuretic peptide to those in whom echocardiography would be expected to meaningfully affect outcome [16]. In our current study, our team has accepted all the prescribed echocardiographies for COVID-19 patients during the study period, except one.

Median LVEF of our patients was 62 (IQR 60-70), and none of our patients had severe LV systolic dysfunction. Three patients have been found to have mild LV systolic dysfunction, with LVEF between 40 and 50\%. One patient was known to have ischemic heart disease, a second patient had suffered from anterior and apical ST-myocardial 
TABLE 1: Clinical and imaging characteristics of the patients included.

\begin{tabular}{|c|c|c|c|c|}
\hline \multirow[b]{2}{*}{ Characteristics } & \multirow[b]{2}{*}{$\begin{array}{c}\text { All TTE/TEE } \\
\text { patients }(n=56)\end{array}$} & \multicolumn{2}{|c|}{ According to treatment change } & \multirow[b]{2}{*}{$\begin{array}{c}P \\
\text { value }\end{array}$} \\
\hline & & $\begin{array}{c}\text { TTE/TEE patients with treatment } \\
\text { change induced by the TTE } N=9 \\
(16 \%)\end{array}$ & $\begin{array}{l}\text { TTE/TEE patients without } \\
\text { treatment change induced by the } \\
\text { TTE } N=47(84 \%)\end{array}$ & \\
\hline Age, median (IQR), $y$ & $66(60.5-74)$ & $64(59-74)$ & $67(62-74)$ & 0.78 \\
\hline Female, $N(\%)$ & $18(32.1)$ & $3(33.3)$ & $15(31.9)$ & \multirow[t]{2}{*}{0.93} \\
\hline COVID-19 diagnosis, $N(\%)$ & {$[1]: 4(7.1)$} & [1]: 0 & [1]: $4(8.5)$ & \\
\hline [1] positive nasopharyngeal swab & {$[1+2]: 25(44.6)$} & {$[1+2]: 5(55.6)$} & {$[1+2]: 20(42.6)$} & \multirow{3}{*}{0.75} \\
\hline [2] typical chest CT scan & [2]: $22(39.3)$ & {$[2]: 3(33.3)$} & [2]: $19(40.4)$ & \\
\hline [3] atypical pneumonia on CTscan & {$[3]: 5(8.9)$} & [3]: $1(11.1)$ & {$[3]: 4(8.5)$} & \\
\hline Inaugural symptoms $\$, N(\%)$ & $\begin{array}{c}\text { Neuro.: } 16(28.6) \\
\text { Resp.: } 35(62.5) \\
\text { Viral: } 3(5.3) \\
\text { COVID typical: } 2 \\
\quad(3.6)\end{array}$ & $\begin{array}{c}\text { Neuro.: } 2(22.2) \\
\text { Resp.: } 6(66.7) \\
\text { Viral: } 1(11.1) \\
\text { COVID typical: } 0\end{array}$ & $\begin{array}{c}\text { Neuro.: } 14(29.8) \\
\text { Resp.: } 29(61.7) \\
\text { Viral: } 2(4.3) \\
\text { COVID typical: } 2(4.3)\end{array}$ & 0.75 \\
\hline $\begin{array}{l}\text { Symptoms' severity, } N(\%) \\
\text { [1] no need for oxygen } \\
\text { [2] need for oxygen therapy } \\
\text { [3] intubated }\end{array}$ & $\begin{array}{c}\text { [1]: } 9(16.1) \\
\text { [2]: } 14(25) \\
{[3]: 33(58.9)}\end{array}$ & $\begin{array}{c}{[1]: 0} \\
{[2]: 3(33.3)} \\
3: 6(66.7)\end{array}$ & $\begin{array}{l}{[1]: 9(19.1)} \\
{[2]: 11(23.4)} \\
{[2]: 27(57.5)}\end{array}$ & 0.35 \\
\hline Body area, median (IQR), $\mathrm{m}^{2}$ & $1.895(1.83-1.97)$ & $1.92(1.83-2.06)$ & $1.89(1.83-1.97)$ & 0.35 \\
\hline BMI, median (IQR), Kg/m² & $28.7(25.30-30.15)$ & $29.6(29.1-31.3)$ & $28.1(24.8-29.8)$ & 0.08 \\
\hline Diabetes, $N(\%)$ & $24(43.6)$ & $3(33.3)$ & $21(45.7)$ & 0.50 \\
\hline Hypertension, $N(\%)$ & $35(63.6)$ & $7(77.8)$ & $18(60.9)$ & 0.34 \\
\hline Smoking, $N(\%)$ & $4(7.7 \%)$ & 0 & $4(8.9)$ & 0.41 \\
\hline Alcohol, $N(\%)$ & $4(7.8)$ & 0 & $4(9.1)$ & 0.41 \\
\hline $\begin{array}{l}\text { Known cardiovascular disease, } \\
N(\%) \\
\text { Evolution }^{\#}, N(\%)\end{array}$ & $16(28.6)$ & $2(22.2)$ & $14(29.8)$ & 0.65 \\
\hline $\begin{array}{l}1 \text { discharge (home or } \\
\text { rehabilitation) }\end{array}$ & $1: 20(35.7)$ & $1: 1(11.1)$ & $1: 19(40.4)$ & \multirow{3}{*}{0.24} \\
\hline 2 deceased & $2: 5(8.9)$ & $2: 1(11.1)$ & $2: 4(8.5)$ & \\
\hline $\begin{array}{l}3 \text { still in hospital at the time of } \\
\text { writing }\end{array}$ & $3: 31(55.4)$ & $3: 7(77.8)$ & $3: 24(51.1)$ & \\
\hline Patients with TTE/TEE, $N(\%)$ & $\begin{array}{l}\text { TTE: } 50(87.5) \\
\text { TTE + TEE: } 5 \\
(10.7) \\
\text { TEE: } 1(1.8)\end{array}$ & $\begin{array}{c}\text { TTE: } 7(77.8) \\
\text { TTE + TEE: } 2(22.2) \\
\text { TEE: } 0\end{array}$ & $\begin{array}{l}\text { TTE: } 42(89.4) \\
\text { TTE + TEE: } 4(8.5) \\
\text { TEE: } 1(2.1)\end{array}$ & 0.44 \\
\hline Indications for TTE/T & $1: 9(16.1)$ & $1: 0$ & $1: 9(19.15)$ & \multirow{13}{*}{0.18} \\
\hline 1 pulmonary embolism: suspicion & $1+2+5: 6(10.7)$ & $1+2+5:(11.1)$ & $1+2+5: 5(10.6)$ & \\
\hline $\begin{array}{l}1 \text { pulmonary embolism: suspicion } \\
\text { or evaluation/pulmonary pressures }\end{array}$ & $1+4+6: 1(1.8)$ & $1+4+6: 0$ & $1+4+6: 1(2.1)$ & \\
\hline or evaluation/pulmonary pressures & $1+5+6: 1(1.8)$ & $1+5+6: 1(11.1)$ & $1+5+6: 0$ & \\
\hline 2 left ventricular flllng pressures & $2: 6(10.7)$ & $2: 3(33.3)$ & $2: 3(6.4)$ & \\
\hline 3 troponin $T$ hs elevation & $1+2+5+7: 1(1.8)$ & $1+2+5+7: 0$ & $1+2+5+7: 1(2.1)$ & \\
\hline $\begin{array}{l}4 \text { exclude infectious endocarditis } \\
5 \text { left ventricular systolic function }\end{array}$ & $3: 2(3.6)$ & $3: 1(11.1)$ & $3: 1(2.1)$ & \\
\hline $\begin{array}{l}5 \text { leit ventricular systolic runction/ } \\
\text { hemodynamic }\end{array}$ & $4: 4(7.14)$ & $4: 0$ & $4: 4(8.5)$ & \\
\hline $\begin{array}{l}\text { hemodynamic } \\
6 \text { ischemic stroke: seek cardiac }\end{array}$ & $5: 11(19.6)$ & $5: 2(22.2)$ & $5: 9(19.15)$ & \\
\hline 6 1schemic stroke: seek cardiac & $1+5: 2(3.6)$ & $1+5: 0$ & $1+5: 2(4.3)$ & \\
\hline source of embolism & $6: 8(14.3)$ & $6: 1(11.1)$ & $6: 7(14.9)$ & \\
\hline 7 other (valvular prosthesis, renal & $6+7: 1(1.8)$ & $6+7: 0$ & $6+7: 1(2.1)$ & \\
\hline & $7: 4(7.14)$ & $7: 0$ & $7: 4(8.5)$ & \\
\hline CT/TEE documented PE, $N(\%)$ & $\begin{array}{c}\text { Yes: } 10(17.9) \\
\text { No: } 16(28.6) \\
\text { Not assessed: } 30 \\
\quad(53.5)\end{array}$ & $\begin{array}{c}\text { Yes: } 2(22.2) \\
\text { No: } 3(33.3) \\
\text { Not assessed: } 4(44.4)\end{array}$ & $\begin{array}{c}\text { Yes: } 8(17.0) \\
\text { No: } 13(27.7) \\
\text { Not assessed: } 26(55.3)\end{array}$ & 0.83 \\
\hline LVEF $^{* *}$ on TTE & $>50 \%: 51(91.1)$ & >50\%: 8 (88.9) & $>50 \%: 43(91.5)$ & \multirow{2}{*}{ NS } \\
\hline 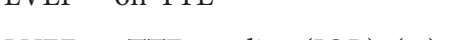 & $40-50 \%: 5(8.9)$ & 40-50\%: 1 (11.1) & $40-50 \%: 4(8.5)$ & \\
\hline LVEF on TTE, median (IQR), (\%) & $62(60-70)$ & $64(52-76)$ & $62(60-70)$ & 0.90 \\
\hline $\begin{array}{l}\text { E/A (transmitral flow), median } \\
\text { (IQR) }\end{array}$ & $0.8(0.7-1)$ & $0.75(0.6-1.0)$ & $0.8(0.8-0.9)$ & 0.50 \\
\hline E/Ea on TTE, median (IQR) & $8.8(6.5-11.8)$ & $8.5(6.7-10.4)$ & $8.9(6.5-11.8)$ & 0.83 \\
\hline
\end{tabular}


TABLE 1: Continued.

\begin{tabular}{|c|c|c|c|c|}
\hline \multirow[b]{2}{*}{ Characteristics } & \multirow[b]{2}{*}{$\begin{array}{c}\text { All TTE/TEE } \\
\text { patients }(n=56)\end{array}$} & \multicolumn{2}{|c|}{ According to treatment change } & \multirow[b]{2}{*}{$\begin{array}{c}P \\
\text { value }\end{array}$} \\
\hline & & $\begin{array}{c}\text { TTE/TEE patients with treatment } \\
\text { change induced by the TTE } N=9 \\
(16 \%)\end{array}$ & $\begin{array}{l}\text { TTE/TEE patients without } \\
\text { treatment change induced by the } \\
\text { TTE } N=47(84 \%)\end{array}$ & \\
\hline $\begin{array}{l}\text { Systolic PAP, median (IQR), } \\
\text { mmHg }\end{array}$ & $38(31-43)$ & $40(38-55)$ & $36(30-43)$ & 0.0549 \\
\hline Pericardial effusion, $N(\%)$ & $2(3.6)$ & 0 & $2(4.3)$ & 0.53 \\
\hline $\begin{array}{l}\text { Cardiac thrombus (TTE or TEE), } \\
N(\%)\end{array}$ & $3(5.4)$ & $3(33.3)$ & 0 & $\leq 0.001$ \\
\hline
\end{tabular}

IQR: interquartile range; $y$ : years, CT: computed tomography; PE: pulmonary embolism; PAPs: echocardiographic systolic pulmonary arterial pressure. ${ }^{\$}$ Inaugural symptoms: Neuro.: neurological; Resp.: respiratory; Viral: nonspecific viral syndrome; COVID: typical signs and symptoms of COVID-19. ${ }^{\#}$ Evolution: 1 discharge (to home or rehabilitation); 2 deceased; 3 still in hospital on 17/04/2020 (at the date of writing this manuscript). ${ }^{* *}$ None of the patients had LVEF $<40 \%$ in this study.

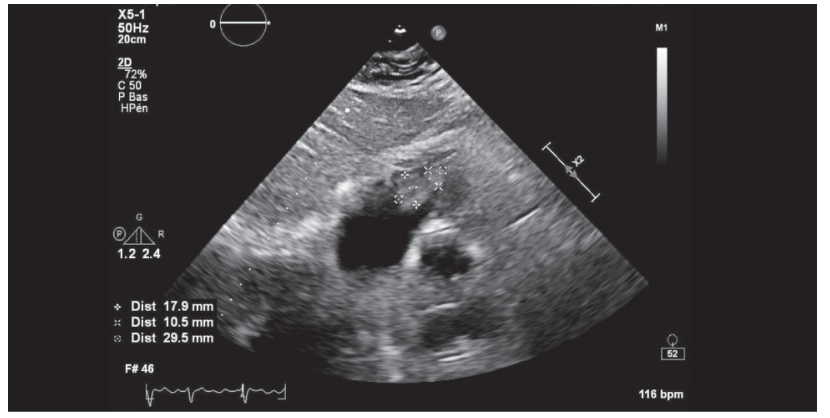

(a)

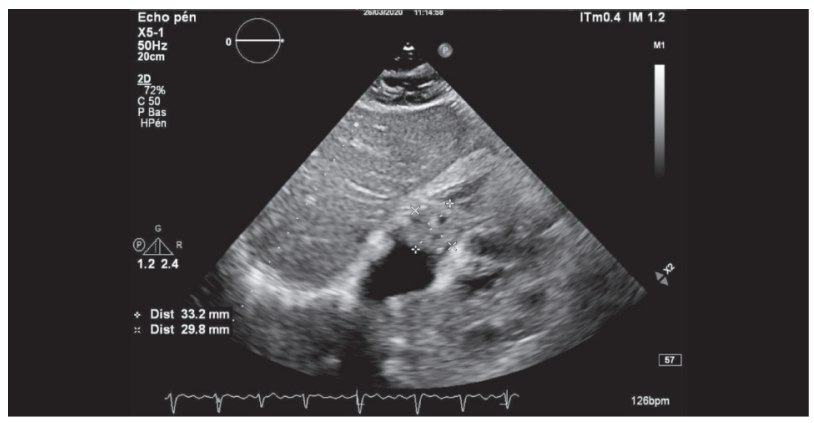

(b)

Figure 1: TTE, subcostal view, showing a right atrial thrombus in a COVID-19 suspected patient (deceased after discharge). The thrombus was prolapsing in the right ventricle in diastole.

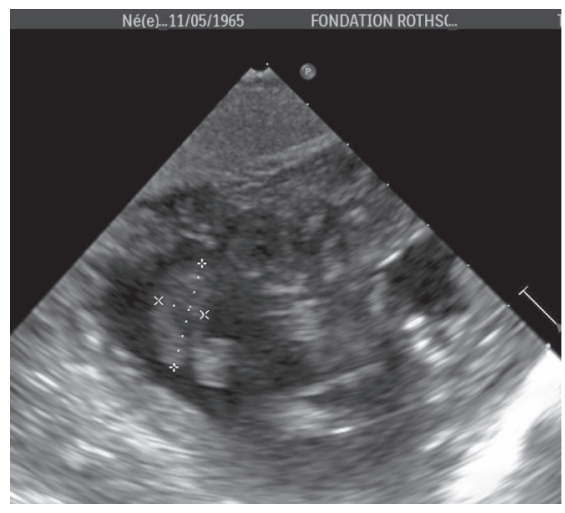

(a)

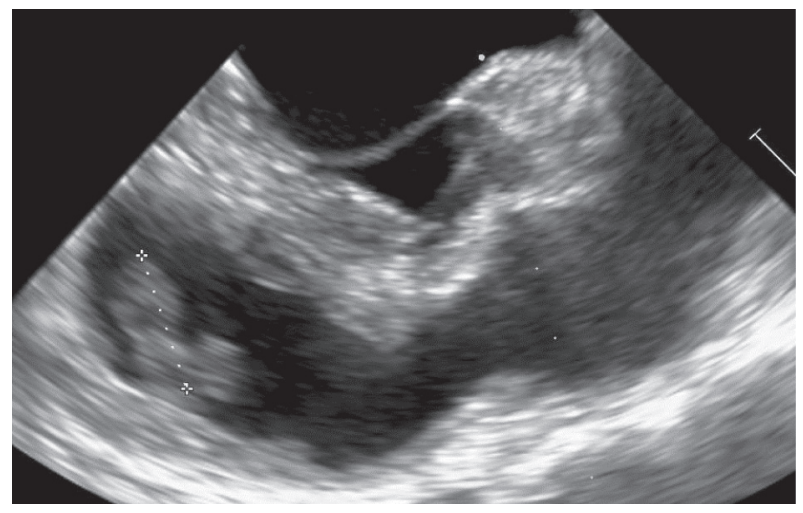

(b)

FIGURE 2: Voluminous and heterogeneous right atrial thrombus seen on TTE (a) and TEE (b). The patient had thrombolysis for severe pulmonary embolism.

infarction during the current hospitalization, and a third patient was admitted for multiple ischemic strokes. In this third patient, TTE at admission was suggestive of myocardial stunning. However, the poor outcome of this patient did not allow confirmation of the diagnosis. ECG, Troponin, and NT-pro BNP at admission were normal.
Echocardiographic LV filling pressures are another important issue in the management of patients with respiratory distress since cardiac participation is often questioned. In this study, when considering the cutoff of average $E / e^{\prime}>14$ for diastolic dysfunction in patients with normal LVEF [17], only 4 patients were identified. The first was a 74- 


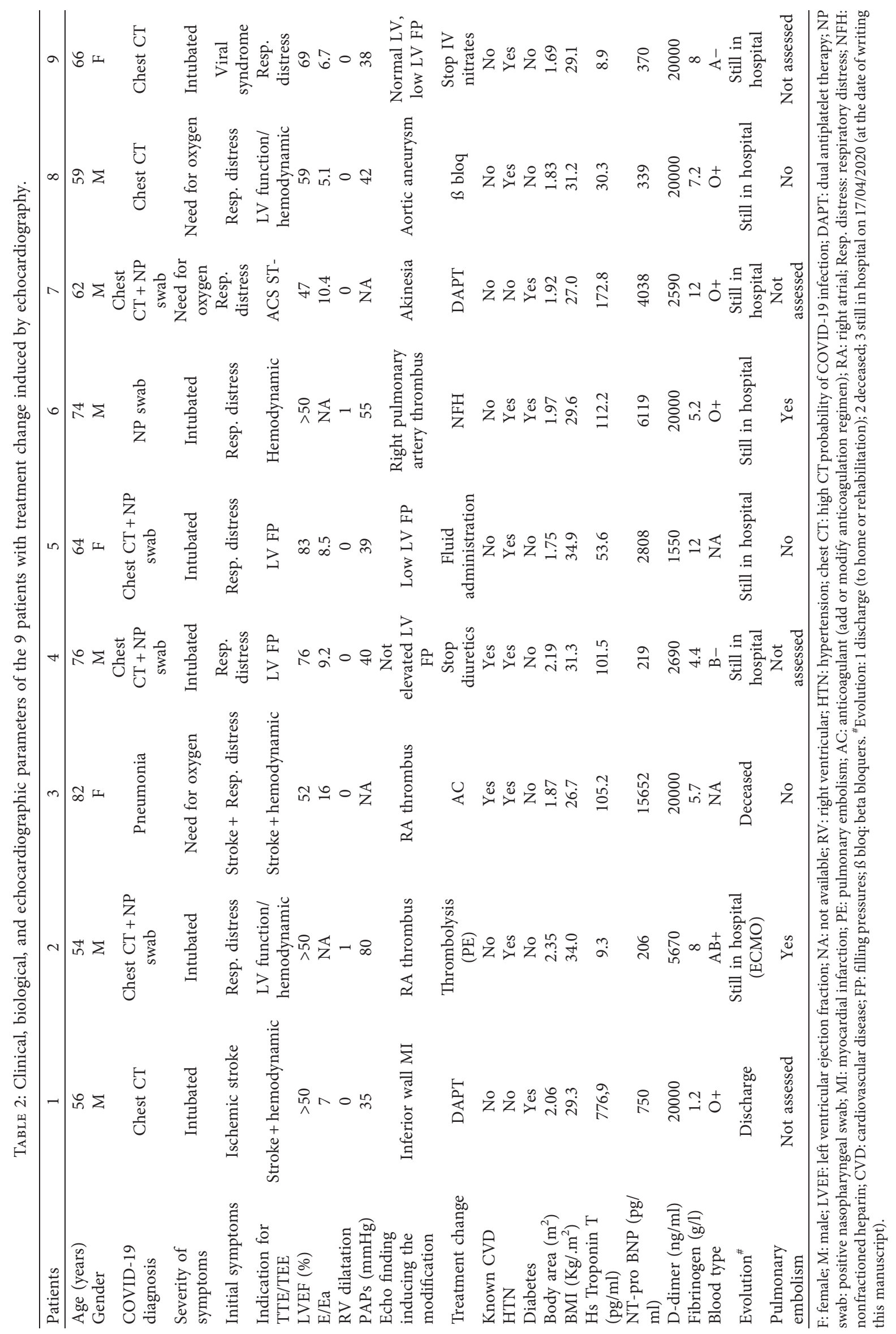


TABLE 3: Biological characteristics of the patients included.

\begin{tabular}{|c|c|c|c|c|}
\hline \multirow[b]{2}{*}{ Characteristics } & \multirow[b]{2}{*}{$\begin{array}{c}\text { All TTE/TEE } \\
\text { patients }(n=56)\end{array}$} & \multicolumn{2}{|c|}{ According to treatment change } & \multirow[b]{2}{*}{$\begin{array}{c}P \\
\text { value }\end{array}$} \\
\hline & & $\begin{array}{c}\text { TTE/TEE patients with treatment } \\
\text { change induced by the TTE } N=9 \\
(16 \%)\end{array}$ & $\begin{array}{l}\text { TTE/TEE patients without treatment } \\
\text { change induced by the TTE } N=47(84 \%)\end{array}$ & \\
\hline $\begin{array}{l}\text { Creatinine, median } \\
\text { (IQR), } \mu \mathrm{mol} / \mathrm{l}\end{array}$ & $80(58.5-124)$ & $90(77-126)$ & $74(58-122)$ & 0.4961 \\
\hline $\begin{array}{l}\text { Hs Troponin T, median } \\
\text { (IQR), pg/ml }\end{array}$ & $21(11.8-57)$ & $101.5(30.3-111.2)$ & $20.3(11.6-43.5)$ & 0.0506 \\
\hline $\begin{array}{l}\text { NT-pro BNP, median } \\
\text { (IQR), pg/ml }\end{array}$ & $628(177-2530)$ & $750(339-4038)$ & $552.5(155.5-2138.5)$ & 0.3197 \\
\hline $\begin{array}{l}\text { D-dimer, median } \\
\text { (IQR), ng/ml }\end{array}$ & $2970(1130-11620)$ & $20000(2690-20000)$ & $2770(1350-9330)$ & 0.0142 \\
\hline \multirow[t]{2}{*}{$\begin{array}{l}\text { Fibrinogen, median } \\
\text { (IQR), g/l }\end{array}$} & $7.84(5.72-10.2)$ & $7.15(5.17-7.95)$ & $8.64(5.89-10.2)$ & 0.4111 \\
\hline & $\begin{array}{c}\text { O+: } 17(46.0) \\
\text { A+: } 9(24.3)\end{array}$ & $\begin{array}{c}\mathrm{O}+: 4(57.1) \\
\mathrm{A}+: 0\end{array}$ & $\begin{array}{c}\text { O+: } 13(43.4) \\
A+: 9(30.0)\end{array}$ & \\
\hline \multirow[t]{3}{*}{ Blood type, $\mathrm{N}(\%)$} & $\mathrm{A}-: 2(5.4)$ & A-: $1(14.3)$ & A-: $1(3.3)$ & 0.2900 \\
\hline & $\mathrm{AB}+: 2(5.4)$ & $\mathrm{AB}+: 1(14.3)$ & $\mathrm{AB}+: 1(3.3)$ & \\
\hline & $\mathrm{B}+: 7(18.9)$ & $\mathrm{B}+: 1(14.3)$ & $\mathrm{B}+: 6(20.0)$ & \\
\hline
\end{tabular}

NT-pro BNP:N-terminal pro B natriuretic peptide; Hs Troponin T: high-sensitivity cardiac Troponin T.

year-old woman admitted for COVID-19 related respiratory distress. She had persistent atrial fibrillation and has been hospitalized in another hospital 4 weeks before for heart failure with preserved LVEF. The dosage of diuretics has been increased during her stay in our hospital, but we did not include this patient in the "treatment change" group. The second was a 56-year-old man admitted for multiple ischemic strokes. ECG was normal, high-sensitivity (hs) Troponin T was $17.5 \mathrm{pg} / \mathrm{ml}$, and NT-pro-BNP was $99.0 \mathrm{pg} /$ $\mathrm{ml}$. He was discharged after 9 days, without complication. The third was an 82-year-old woman with documented right atrial thrombus (see Figure 2(b)). She has unfortunately deceased in rehabilitation. The last patient was a 74-year-old woman admitted for COVID-19 related respiratory distress. She had CT-documented pulmonary embolism, abnormal ECG, hs Troponin T of $11.8 \mathrm{pg} / \mathrm{ml}$, and NT-pro BNP of 1569 $\mathrm{pg} / \mathrm{ml}$. She has been discharged on day twelve.

Indeed, LVEF as evaluated by two-dimensional echocardiography is not sufficient alone for the diagnosis and the treatment of acute heart failure. The pitfalls of this method have been largely highlighted [18]. This is why our team of cardiologists have decided to perform systematically mitral pulsed Doppler imaging and tissue Doppler imaging, before having guidelines and recommendations in the particular setting of COVID-19, so as to approach left ventricular filling pressures and help the practitioners in fluid management of their patients (vascular filling versus diuretics).

4.4. Cardiac Injury. Cardiac injury is frequent in COVID-19 patients and associated with a higher risk of in-hospital mortality [5]. Recently, COVID-19 related myocarditis, confirmed by multimodality imaging, was described in a 21year-old female patient [19]. Triggered COVID-19 Takotsubo syndrome has also been reported in an 83-year-old woman [20]. Acute myocarditis presenting as a reverse Takotsubo syndrome was also reported in a COVID-19, 43- year-old woman, with both cardiac magnetic resonance imaging and endomyocardial biopsy characterization [21].

There are various mechanisms for hs Troponin elevation in COVID-19 patients [22]. For now, the American College of Cardiology does not recommend Troponin measurement, unless the diagnosis of acute myocardial infarction is being considered [16]. However, it could be useful in determining high-risk patients, for more intensive surveillance and support [23]. In our study, only 3 patients did not have at least one Troponin measurement. The median hs Troponin $\mathrm{T}$ was 101.5 (IQR 30.3-111.2) in patients with a treatment change in relation to echocardiography and 20.3 (IQR 11.6-43.5) in the others. The difference did nearly reach statistical significance $(P=0.0506)$.

4.5. Body Mass Index. The median BMI of the whole series was 28.7 (25.30-30.15) (see Table 1), and there was a trend to a higher BMI in patients with more severe illness, with mean BMI (SD) of $26.65 \mathrm{Kg} / \mathrm{m}^{2}(4.52)$ in the patients who did not require oxygen therapy $(N=9)$, mean BMI of $27.13(3.60)$ in the patients requiring oxygen therapy $(N=14)$, and mean BMI of $28.88 \mathrm{Kg} / \mathrm{m}^{2}$ (4.35) in the intubated patients. However, these last analyses were exploratory, since our study was not designed for that, and the difference did not reach statistical significance $(P=0.2374)$.

4.6. Observers' Safety. In our experience, PPE has proved to be effective. Among the two cardiologists and the three nurses who participated in the study, none had COVID-19 symptoms. Furthermore, all the five were tested in June 2020. Their serological investigations were negative.

While writing this manuscript, a helpful framework for addressing cardiovascular complications associated with COVID-19 has been published [23]. It recommends Troponin evaluation in all confirmed COVID-19 patients who require hospitalization. Our results are in line with it since 
only $7 \%$ of our patients did not have Troponin evaluation. Furthermore, our results confirm the usefulness of TTE in the management of COVID-19 patients with elevated cardiac biomarkers and D-dimer. Point-of-care ultrasound (POCUS) was not yet developed in our institution. However, echocardiographies were fast echos in all the patients, unless an abnormal finding was documented. In this case, the echocardiography was complete.

4.7. Study Limitations. Our study has a modest sample size to assess the impact of echocardiography on COVID-19 patients' management and there was no power calculation. However, we are still including patients.

Initial data on heart consequences of this dramatic disease have been rapidly published since February 2020. However, we believe that this study is, as of this date, the first study describing a population of COVID-19 patients who underwent echocardiography and evaluating the impact of this echocardiography on the management of these patients, in correlation with clinical, biological, and echocardiographic data.

Finally, how was the treatment change directly prompted by echocardiography could be questioned. However, this study was exclusively driven by practical routine clinical practice. Hence, echocardiography was performed at the discretion of the practitioner in charge of the patient, in order to respond to one or more of his unresolved questions regarding patient's cardiac condition. Subsequent decisions regarding the treatment were taken jointly by the practitioner and the cardiologist, depending on the results of the echocardiography.

\section{Conclusion}

The majority of our patients had preserved LVEF and normal LV filling pressures while pulmonary embolism was documented in $17.9 \%$ of cases.

We found $16 \%$ of treatment change induced by echocardiography, and D-dimer and Troponin levels were predictive of this treatment change.

We believe that these findings may be useful in general practice for selecting the patients who would more benefit from echocardiography, especially in the hospitals and the countries where cardiologic and echocardiographic resources are rare, in this uncertain period, and the fear of a second wave.

Finally, none of the practitioners involved in the study had symptoms of COVID-19 and all their serological investigations were negative.

Further research on a larger scale would be needed to confirm our findings.

\section{Abbreviations}

COVID- Coronavirus disease 2019

19:

CRF: $\quad$ Case fatality rate

CVD: Cardiovascular disease

E/e':
Mitral valve $E$ velocity divided by mitral annular e' velocity

HTN: Hypertension

Hs High-sensitivity Troponin

Troponin:

LVEF: Left ventricular ejection fraction

PAPs: Pulmonary arterial systolic pressure

PPE: $\quad$ Personal protective equipment

TEE: Transesophageal echocardiography

TTE: Transthoracic echocardiography.

\section{Data Availability}

The underlying data may be obtained from the corresponding author.

\section{Disclosure}

The authors declare that they have not published, posted, or submitted any related papers from this study.

\section{Conflicts of Interest}

The authors do not report any relevant conflicts of interest in relation to this article.

\section{Acknowledgments}

All nurses, anesthesiologists, intensivists, neurologists, and infectious disease specialists working hard in our hospital and our country in this difficult period are greatly acknowledged for their massive efforts and daily care for critically ill patients suffering from SARS-CoV-2 infection. Our nurses Malika Allaoui, Julien Aubert, Gisèle Chevalier, and Aurélien Guillot are also acknowledged, for their participation in this study.

\section{References}

[1] E. Dong, H. Du, and L. Gardner, "An interactive web-based dashboard to track COVID-19 in real time," The Lancet Infectious Diseases, vol. 20, no. 5, pp. 533-534, 2020.

[2] B. Li, J. Yang, F. Zhao et al., "Prevalence and impact of cardiovascular metabolic diseases on COVID-19 in China," Clinical Research in Cardiology, vol. 109, no. 5, pp. 531-538, 2020.

[3] Y.-Y. Zheng, Y.-T. Ma, J.-Y. Zhang, and X. Xie, "COVID-19 and the cardiovascular system," Nature Reviews Cardiology, vol. 17, no. 5, pp. 259-260, 2020.

[4] F. Zhou, T. Yu, R. Du et al., "Clinical course and risk factors for mortality of adult inpatients with COVID-19 in Wuhan, China: a retrospective cohort study," The Lancet, vol. 395, no. 10229, pp. 1054-1062, 2020.

[5] S. Shi, M. Qin, B. Shen et al., "Association of cardiac injury with mortality in hospitalized patients with COVID-19 in wuhan, China," JAMA Cardiology, vol. 5, no. 7, p. 802, 2020.

[6] Z. Wu and J. M. McGoogan, "Characteristics of and important lessons from the coronavirus disease 2019 (COVID19) outbreak in China," JAMA, vol. 323, no. 13, pp. 1239-1242, 2020. 
[7] E. Driggin, M. V. Madhavan, B. Bikdeli et al., "Cardiovascular considerations for patients, health care workers, and health systems during the COVID-19 pandemic," Journal of the American College of Cardiology, vol. 75, no. 18, pp. 2352-2371, 2020.

[8] N. Benyounes, S. Lang, O. Gout, Y. Ancédy, A. Etienney, and A. Cohen, "Could quantitative longitudinal peak systolic strain help in the detection of left ventricular wall motion abnormalities in our daily echocardiographic practice?," Archives of Cardiovascular Diseases, vol. 109, no. 10, pp. 533-541, 2016.

[9] N. Benyounes, S. Lang, L. Soulat-Dufour et al., "Can global longitudinal strain predict reduced left ventricular ejection fraction in daily echocardiographic practice?," Archives of Cardiovascular Diseases, vol. 108, no. 1, pp. 50-56, 2015.

[10] N. Benyounes, C. Van Der Vynckt, T. Tibi et al., "Left ventricular end diastolic volume and ejection fraction calculation: correlation between three echocardiographic methods," Cardiology Research and Practice, vol. 2020, p. 1, 2020.

[11] J. N. Kirkpatrick, C. Mitchell, C. Taub, S. Kort, J. Hung, and M. Swaminathan, "ASE statement on protection of patients and echocardiography Service Providers during the 2019 novel coronavirus outbreak," Journal of the American College of Cardiology, vol. 75, no. 24, p. 3078, 2020.

[12] G. B. Danzi, M. Loffi, G. Galeazzi, and E. Gherbesi, "Acute pulmonary embolism and COVID-19 pneumonia: a random association," European Heart Journal, vol. 41, no. 19, p. 1858, 2020.

[13] N. Tang, D. Li, X. Wang, and Z. Sun, “Abnormal coagulation parameters are associated with poor prognosis in patients with novel coronavirus pneumonia," Journal of Thrombosis and Haemostasis, vol. 18, no. 4, pp. 844-847, 2020.

[14] Y. Zhang, M. Xiao, S. Zhang et al., "Coagulopathy and antiphospholipid antibodies in patients with covid-19," New England Journal of Medicine, vol. 382, no. 17, p. e38, 2020.

[15] S. S. Mitter, S. J. Shah, and J. D. Thomas, "A test in context," Journal of the American College of Cardiology, vol. 69, no. 11, pp. 1451-1464, 2017.

[16] American College of Cardiology, "Troponin and BNP use in COVID-19," 2017.

[17] S. F. Nagueh, O. A. Smiseth, C. P. Appleton et al., "Recommendations for the evaluation of left ventricular diastolic function by echocardiography: an update from the American society of echocardiography and the European association of cardiovascular imaging," Journal of the American Society of Echocardiography, vol. 29, no. 4, pp. 277-314, 2016.

[18] P. Severino, V. Maestrini, M. V. Mariani et al., "Structural and myocardial dysfunction in heart failure beyond ejection fraction," Heart Failure Reviews, vol. 25, no. 1, pp. 9-17, 2020.

[19] I.-C. Birtolo, J. Y. Kim, H. A. Kim, and S. Han, "COVID-19related myocarditis in a 21-year-old female patient," European Heart Journal, vol. 41, no. 19, p. 1859, 2020.

[20] P. Meyer, S. Degrauwe, C. Van Delden, J.-R. Ghadri, and C. Templin, "Typical takotsubo syndrome triggered by SARSCoV-2 infection," European Heart Journal, vol. 41, no. 19, p. $1860,2020$.

[21] S. Sala, G. Peretto, M. Gramegna et al., "Acute myocarditis presenting as a reverse Tako-Tsubo syndrome in a patient with SARS-CoV-2 respiratory infection," European Heart Journal, vol. 41, no. 19, pp. 1861-1862, 2020.

[22] A. R. Chapman, A. Bularga, and N. L. Mills, "High-sensitivity cardiac troponin can Be an ally in the fight against COVID19," Circulation, vol. 41, 2020.
[23] R. S. Loungani, M. R. Rehorn, and L. K. Newby, "A care pathway for the cardiovascular complications of COVID-19: insights from an institutional response," American Heart Journal, vol. 225, pp. 3-9, 2020. 